\title{
Investigation of a neuropsychological screen for chemo-fog
}

\author{
Lea Ann Ouimet • Angela Stewart • \\ Barbara Collins $\cdot$ Dwayne Schindler • \\ Catherine Bielajew
}

Received: 13 June 2011 / Accepted: 26 September 2011/Published online: 14 October 2011

(C) Springer-Verlag 2011

\begin{abstract}
Research on chemotherapy-induced cognitive impairment (the term "chemo-fog" is used by many investigators) supports the occurrence of subtle declines in function for a subset of recipients. Identification of vulnerable individuals via comprehensive neuropsychological batteries is complicated due to their lack of clinical utility and increased risk of misclassification. The goal of this paper was to evaluate the ability of a reduced battery to detect chemotherapy-related cognitive impairments. Data from our previous study (Ouimet et al. J Clin Exp Neuropsychol 31:73-89, 2009) were used to compare a comprehensive neuropsychological test battery comprising 23 tests with a reduced battery consisting of a subset of nine tests. A standardized regression-based approach revealed that a comparable numbers of participants were identified by both batteries, suggesting that individuals vulnerable to chemotherapy-induced cognitive impairment can be identified by a more selective battery. Further work is needed to clarify the neuropsychological tests most sensitive to detecting impairments associated with chemotherapy so that assessment batteries can be limited to these tests.
\end{abstract}

Keywords Breast cancer - Chemotherapy - Cognitive impairment - Neuropsychological assessment .

Misdiagnosis

L. A. Ouimet $(\bowtie) \cdot$ D. Schindler · C. Bielajew

School of Psychology, University of Ottawa,

136 Jean Jacques Lussier, Ottawa, ON K1N 6N5, Canada

e-mail: louim095@uottawa.ca

\author{
A. Stewart \\ Royal Ottawa Health Care Group, Ottawa, ON, Canada \\ B. Collins \\ Ottawa Hospital, Civic Campus, Ottawa, ON, Canada
}

\section{Introduction}

Breast cancer strikes approximately one in nine women each year in North America [2,3] and the majority will receive some form of chemotherapy as part of their treatment regimen. In the last few years, there has been increasing evidence to suggest that a subset of these women suffer from chemotherapy-related cognitive impairments that primarily affect memory, attention, concentration, and perseverance [4-16].

While from a statistical standpoint the effects have generally been small; from a clinical perspective, the degree of impairment noted in both self-report research and those using objective measurement might reasonably be expected to have major impacts on quality of life. In fact, deleterious impact of cognitive decline from chemotherapy on quality of life in breast cancer patients has been reported by various researchers [7, 13, 16-24].

Given the incidence of breast cancer, and the standard inclusion of some form of chemotherapy as part of the treatment regimen, if quality of life is negatively impacted, then the identification of chemotherapy-related cognitive impairments becomes an issue of clinical relevance. Unfortunately, various issues in the area of neuropsychological assessment complicate this process, making it difficult to accurately identify the subgroup of women affected.

Although there is a substantial literature on test theory and professional guidelines for the selection of individual tests, a salient issue affecting neuropsychology is the lack of theory underlying the optimal composition of a neuropsychological test battery. As a result, there is wide variability in the choice of tests used to measure various cognitive domains, making it difficult to accurately identify the areas of functioning that are most compromised 
following chemotherapy treatment. Compounding the problem is the very nature of cognitive functions; they are complex and overlapping, making it a considerable challenge to choose tests that are as "clean" as possible (i.e. measuring one specific construct).

Given these limitations, it is not surprising that there are few professional guidelines or policies available to instruct clinicians on the most effective and reliable means of constructing a neuropsychological test battery. As a result, tests may be chosen as a function of availability, tradition, familiarity, or personal preference rather than evidence of reliability, validity, or statistical support.

Another important consideration is the historical impetus for the development of neuropsychological tests, which was in fact, to assist in diagnostics, not in the prediction of outcome. However, assessments are used commonly today for aiding in the determination of expected future performance, achievement, or recovery and to evaluate whether or not cognitive changes occur over time. Whether or not or to what extent neuropsychological tests designed for diagnostics retain their validity and reliability when used for predictive purposes can only be answered through continued research on these aspects.

There is also some controversy regarding the choice of fixed versus flexible test batteries [25]. A fixed battery refers to an assembled, normed set of tests, in contrast to flexible batteries, which are chosen and adjusted based on a dynamic hypothesis regarding the nature of the presenting problem, as well as the patient's performance throughout the assessment. A third approach, the intermediate position [26], refers to a core battery that is supplemented by tests relevant to the diagnosis of a particular population who show specific deficits (e.g., Alzheimer's patients) as well as the patient's performance.

A related issue that further complicates matters is the number of tests used in any given neuropsychological test battery. From a research perspective, as the number of tests multiplies, so does the likelihood of redundancy and multicollinearity. Adding more tests also reduces power and the probability of finding statistical significance, thereby escalating Type II errors. Practically, a longer battery may decrease participation overall, and raise attrition in longitudinal studies. But perhaps the most critical disadvantage of a large battery, from both a research and clinical perspective, is the risk of misclassification.

Viewed from a purely clinical standpoint, a larger battery increases the time required for administration and introduces potentially conflicting explanations for poor test performance such as fatigue, loss of attention and focus, and decreased motivation.

Although all of these issues pertain to the area of breast cancer research, one that is of particular clinical relevance in this area is the size of the battery used. They typically consist of a large number of tests, and while this may be appropriate in a research context, they have limited clinical utility because they are too big to be of practical use for screening in health care settings. Consequently, the objective of this paper is to use the data obtained in our previous study [1] to aid in the construction of a smaller neuropsychological test battery for screening "chemofog". In the earlier study, we found that a subset of participants in both the chemotherapy and hormonal groups experienced a significant decline in function (greater than two standard deviations) on two or more tests in a neuropsychological test battery.

Specifically, this paper compares a neuropsychological test battery comprising 23 tests representing the major cognitive domains (executive, language, motor, processing speed, verbal learning and memory, visual learning and memory, visuospatial, and working memory) with a subset of nine tests, in order to evaluate the sensitivity of the reduced battery to chemotherapyinduced cognitive impairments. For the remainder of this paper, our original 2009 data and results [1] will be referred to as study 1 and the current paper will be referred to as study 2 .

\section{Model}

To determine if the reduced neuropsychological test battery was sensitive in detecting impaired performance, standard regression-based analyses (SRB) [27] were applied to assess whether the individuals in the hormonal and chemotherapy groups show a significant decline across time. SRB analyses were chosen as opposed to the reliable change index because SRB take into account regression and individual practice effects. This method also allows for the inclusion of covariates.

Whereas, the previous study consisted of two separate sets of SRB analyses using the means and standard deviations of both a healthy control group and a hormonal group as an estimate for evaluating measurement error and practice effects, the current study used only the healthy control group.

This decision was based on an evaluation of the results obtained in the first study in that the differences between the chemotherapy and hormonal groups were less than the differences between either of these groups and a healthy control reference. As a result, the use of the hormonal group as a basis for comparison in this context is limited because of the risk of underestimating cognitive decline, thereby increasing the risk of misclassification. To minimize this risk, the healthy control group was used for comparison. 


\section{Method}

Participants

Data were obtained from 123 stage I or II breast cancer patients who were recruited as part of a larger longitudinal study conducted out of the Ottawa Regional Cancer Centre to investigate the neuropsychological effects of adjuvant chemotherapy. All participants had undergone mastectomy or lumpectomy and were receiving chemotherapy (with or without hormonal treatment, $n=49$ ) or hormonal treatment alone $(n=46)$. Details regarding disease stage, treatment regimens, and number of chemotherapy cycles can be found in Table 1 .

In addition, a healthy control group $(n=28)$ was recruited through advertisements posted on hospital bulletin boards. This group was included to examine practice effects and to facilitate evaluation of these effects in the treatment groups as well as to provide a means of evaluating decline from a healthy state. Participants received $\$ 50$ for each test session.

\section{Sample selection}

The sample was limited to postmenopausal women aged 65 years or younger as a means of minimizing the potential confounds of hormonal status and age-related cognitive decline. Fluency in English was required in order to complete the test battery. Exclusion criteria for all groups included a history of previous cancer and chemotherapy or radiation treatment and serious psychiatric disorder (e.g.,

Table 1 Clinical characteristics of treatment groups

\begin{tabular}{llll}
\hline Characteristic & $\begin{array}{l}\text { Chemotherapy } \\
(N=49)\end{array}$ & $\begin{array}{l}\text { Hormonal } \\
(N=46)\end{array}$ & $p$ \\
\hline Stage of disease & & & $<.001$ \\
I & $(n=13)$ & $\begin{array}{l}(n=39) \\
(n=5)\end{array}$ & \\
II & $(n=34)$ & NA & NA \\
III & $(n=2)$ & & \\
Type of chemotherapy & & \\
FEC-100 & $(n=27)$ & & \\
AC/AC-Taxol & $(n=13)$ & & NA \\
CEF & $(n=3)$ & & \\
FAC & $(n=3)$ & & \\
Other & $(n=3)$ & & \\
Number of chemotherapy cycles & & \\
Mean (SD) & $5.69(1.17)$ & & \\
Min/Max & $4-10$ & & \\
\end{tabular}

FEC-100 Flourouracil, epirubicin, cyclophosphamide; $A C$ doxorubicin, cyclophosphamide; AC-Taxol doxorubicin, cyclophosphamide, taxol; $C E F$ cyclophosphamide, epirubicin, fluorouracil; $F A C$ cyclophosphamide, doxorubicin, fluorouracil major depression, schizophrenia), neurological illness, or significant substance abuse due to their potential negative effects on cognitive function. The board of ethics at the Ottawa Hospital approved this study and written informed consent was obtained from all participants.

\section{Measures}

Our original study included a total of 23 psychometrically sound neuropsychological tests representing the major cognitive domains: executive function, language function, motor skills, processing speed, verbal learning and memory, visual learning and memory, visuospatial function, and working memory (please see [1] for a detailed list). Nine subtests were retained for the present paper, including: Trails A and B, FAS (Controlled Oral Word Association Test), Wechsler Adult Intelligence Scale-III Digit Symbol Coding, Symbol Search and Letter-Number Sequencing subtests, California Verbal Learning Test-II Long Delay Free Recall and Long Delay Recognition and Consonant Trigrams. Means and standard deviations for each test are reported in Table 2.

\section{Procedure}

Two commonly used texts on clinical neuropsychological assessment were consulted in order to select the most appropriate tests from the 23 used in our original study for inclusion in the reduced battery: Lezak, Howieson and Loring's Neuropsychological Assessment 4th Edition [28] and Strauss, Sherman and Spreen's A Compendium of Neuropsychological Tests, 3rd Edition [26]. An emphasis was placed on tests purported to measure the functions previously identified as impaired in this population (i.e., language, verbal memory, executive function, processing speed and working memory). In addition, since the objective was to evaluate a battery that could be clinically useful as a screening tool, administration time for individual tests was an important characteristic. Finally, because there are very few studies to date that have examined the sensitivity of individual neuropsychological tests to chemotherapy-related impairments in cognition [10,29], tests were also considered for inclusion if they have been recommended for the evaluation of mild traumatic brain injury because the areas found to be most affected are similar to those noted in chemotherapy-related cognitive impairment [30]. With these resources and considerations in mind, careful evaluation led to the elimination of 14 tests. Table 3 provides an explanation and/or rational for the decision made regarding each test.

\section{Data analysis}

Criterion cut-off scores were calculated using the resampling add-in for Excel. All other analyses were conducted 
Table 2 Means and standard deviations associated with each measure

\begin{tabular}{lllllll}
\hline \multirow{2}{*}{ Test } & \multicolumn{2}{l}{ Baseline } & & & \multicolumn{2}{l}{ Post-chemotherapy } \\
\cline { 2 - 4 } \cline { 5 - 6 } & Healthy control & Hormonal & Chemotherapy & Healthy control & Hormonal & Chemotherapy \\
\hline Trail A & $25.3 \pm 5.4$ & $27.2 \pm 9.1$ & $27.6 \pm 8.2$ & $25.1 \pm 5.4$ & $26.3 \pm 6.8$ & $27.1 \pm 9.4$ \\
Trail B & $65.2 \pm 15.8$ & $70.2 \pm 22.9$ & $69.9 \pm 25.8$ & $59.8 \pm 15.9$ & $70.0 \pm 21.8$ & $67.1 \pm 27.0$ \\
FAS, number of correct words & $43.1 \pm 13.5$ & $37.6 \pm 10.5$ & $41.5 \pm 12.4$ & $44.4 \pm 11.6$ & $39.4 \pm 10.8$ & $40.3 \pm 12.7$ \\
CVLT-II, Long Delay Free Recall & $13.5 \pm 2.1$ & $12.0 \pm 2.6$ & $12.4 \pm 3.0$ & $14.5 \pm 1.6$ & $12.6 \pm 2.5$ & $13.1 \pm 2.5$ \\
CVLT-II, Long Delay Yes-No & $15.2 \pm 1.2$ & $15.0 \pm 1.3$ & $15.2 \pm 1.1$ & $15.5 \pm .9$ & $15.0 \pm 1.3$ & $15.4 \pm 1.0$ \\
WAIS-III, Digit Symbol Coding & $72.3 \pm 11.3$ & $68.9 \pm 11.3$ & $67.7 \pm 12.5$ & $75.6 \pm 10.2$ & $68.7 \pm 11.9$ & $68.2 \pm 13.4$ \\
WAIS-III, Symbol Search & $30.5 \pm 5.9$ & $30.3 \pm 5.0$ & $30.9 \pm 6.6$ & $32.1 \pm 5.3$ & $30.5 \pm 4.9$ & $31.3 \pm 6.9$ \\
WAIS-III, Letter-Number Sequencing & $10.4 \pm 2.6$ & $10.5 \pm 2.2$ & $10.8 \pm 2.8$ & $11.3 \pm 2.5$ & $11.0 \pm 2.3$ & $10.3 \pm 2.6$ \\
CCC, total & $49.6 \pm 5.8$ & $43.0 \pm 7.3$ & $43.4 \pm 7.6$ & $51.3 \pm 5.1$ & $44.7 \pm 7.7$ & $43.1 \pm 8.7$ \\
\hline
\end{tabular}

WAIS-III Wechsler Adult Intelligence Scale-III, CVLT-II California Verbal Learning Test II, CCC Consonant Trigrams

Table 3 Rational for test selection

\begin{tabular}{|c|c|c|}
\hline Test & Status & Rational \\
\hline Trails A and B & Retained & $\begin{array}{l}\text { Shown to be highly sensitive to cognitive deficits in traumatic brain injury (TBI); } \\
\text { minimal practice effects over longer intervals ( } 1 \text { year), quick administration }\end{array}$ \\
\hline FAS, number of correct words & Retained & $\begin{array}{l}\text { High reliability; sensitive to detection of TBI; good measure of verbal abilities; } \\
\text { quick administration }\end{array}$ \\
\hline $\begin{array}{l}\text { CVLT-II, Long Delay Free Recall and Long } \\
\text { Delay Yes/No Recognition }\end{array}$ & Retained & Solid internal consistency, good test-retest reliability \\
\hline Symbol Search and Digit Symbol Coding & Retained & $\begin{array}{l}\text { Low specificity on its own, but solid measure of processing speed when } \\
\text { combined with digit symbol coding; shown to be sensitive to TBI severity; } \\
\text { quick administration }\end{array}$ \\
\hline Letter-Number Sequencing & Retained & Good reliability; sensitive to TBI severity; quick administration \\
\hline $\mathrm{CCC}$, total & Retained & Sensitive to detection of TBI \\
\hline Spatial Span & Removed & Not validated as a measure of visual working memory \\
\hline Logical Memory II & Removed & $\begin{array}{l}\text { Not shown to be measuring a different construct that Logical Memory I; may not } \\
\text { measure delayed memory }\end{array}$ \\
\hline Family Pictures & Removed & $\begin{array}{l}\text { Has not been shown to necessarily capture visual learning; highly influenced by } \\
\text { verbal abilities }\end{array}$ \\
\hline CVLT-II, Trial 1 Free Recall & Removed & More interested in delayed versus immediate recall \\
\hline PASAT $2.4 \mathrm{~s}$, total correct & Removed & $\begin{array}{l}\text { Despite solid psychometrics was very difficult for participants and was often } \\
\text { discontinued; too much missing data as a result; long administration }\end{array}$ \\
\hline $\begin{array}{l}\text { RVLT, Trial } 1 \text { Free Recall } \\
\text { RVLT, Long Delay Free Recall } \\
\text { RVLT, Long Delay Recognition }\end{array}$ & Removed & $\begin{array}{l}\text { More interested in verbal versus visual memory as this has been shown to be } \\
\text { more vulnerable to the effects of chemo-fog }\end{array}$ \\
\hline Block Design & Removed & Good psychometrics but administration time is too long \\
\hline Arithmetic & Removed & $\begin{array}{l}\text { Performance has been shown to depend on math skills as much as Working } \\
\text { Memory; test has been removed from WAIS-IV for this reason }\end{array}$ \\
\hline Digit Span, Forward + Backward & Removed & Not found to be sensitive to degree of severity in TBI \\
\hline WCST, no. of trials, raw score & Removed & $\begin{array}{l}\text { Concerns regarding the aspect of "novelty" required for this test given the test/ } \\
\text { re-test nature of the study }\end{array}$ \\
\hline Boston Naming Test, total & Removed & $\begin{array}{l}\text { Very highly correlated with IQ, education, and age; does not differentiate well } \\
\text { when scores are high }\end{array}$ \\
\hline Grooved Pegboard, combined & Removed & Too much missing data \\
\hline
\end{tabular}

PASAT Paced Auditory Serial Addition Task, WCST Wisconsin Card Sorting Test, WAIS-III Wechsler Adult Intelligence Scale-III, WMS-III Wechsler Memory Scale-III, CVLT-II California Verbal Learning Test II, RVLT Rey Visual Learning Test, FAS Controlled Oral Word Association Test

Sources: Strauss et al. 2006 [26]; Lezak 2004 [28] 
using the Predictive Analytics Software version 18.0, from SPSS Inc.

\section{Standardized regression-based model}

A standardized regression-based model (SRB) [27] was used to evaluate decline in neuropsychological function in the chemotherapy and hormonal groups. This model provided adjustments for measurement error and practice effects and permitted the inclusion of several covariates including age, education, and estimated IQ.

In brief, the SRB approach is used to identify participants who have experienced a significant change in performance across time. For each test, the post-treatment performance is regressed on the baseline and covariates in order to obtain a predicted test score. This value is then subtracted from the obtained score and divided by the standard error of estimate in order to derive a standardized change score. A score greater than \pm 1.64 (falling within the $5 \%$ area at either end of a normal distribution) is indicative of a significant change in performance.

Following the SRB analyses, performance across the nine test variables was summed and participants classified according to the cut-off criterion of significant decline on two or more tests. Once the results were obtained, they were compared to those from study 1 to assess whether the reduced battery was equally able to identify the women who were previously found to be significantly impaired. Three comparisons were made: (1) the percentage of participants who declined on 0 to 9 tests were calculated, (2) the percentage of participants who met the cut-off criterion for each study were calculated, and (3) the percentage of participants who met a more stringent cut-off criterion of significant decline on 4 or more tests in study 1 , and 3 or more tests in study 2 were calculated.

\section{Resampling}

Once individual decline by test was established using SRB techniques, a cut-off criterion to signify an overall decline on the neuropsychological test battery from study 1 [1] was calculated. Rather than using an arbitrary cut-off, a criterion value was derived through the use of resampling with replacement. A dataset was established such that the probability of showing a decline on any one test of the 23 in the battery, simply as a function of chance, was $2.5 \%$ (chosen to represent two standard deviations below the mean). Twenty-three samples were drawn to simulate results on the entire battery for one participant, a procedure that was repeated by the program 65,000 times. Frequencies were then calculated and based on the results, it was determined that the probability of showing significant decline on three or more tests out of 23 , due to chance alone, was slightly $<2 \%$. Thus, a cut-off value of three or more was considered to warrant further clinical investigation. Using the same approach with the reduced battery from study 2 , a cut-off of decline on two or more tests was considered sufficient to warrant further clinical investigation.

These values were chosen as they are indicative of a pattern of decline that would only be seen in slightly $<2 \%$ of the normal population. However, the actual rates of chemotherapy-related cognitive decline are difficult to ascertain; therefore, a more stringent cut-off for decline on four or more tests in study 1 and three or more tests in study 2 is also included for comparison. A pattern of this sort would be expected to occur $<1 \%$ of the time in a normal population.

\section{Results}

Descriptive statistics relating to age, education, estimated IQ at baseline, and test-retest interval are provided in Table 4. There were significant group differences between groups on education and estimated IQ. When these covariates were entered into the model first in the SRB analyses, followed by the post-treatment score, they accounted for a significant proportion of the variance in the Symbol Search $\left(R^{2}=.29, F \Delta(3,24)=3.20, p=.04\right)$ subtest of the WAIS-III.

Figure 1 presents the breakdown of participants in each group who declined on each of 0 to 9 tests based on the reduced battery (left side) and the long battery (right side). This figure illustrates the variability in the obtained results as a function of the number of tests included in the neuropsychological battery.

Figure $2 \mathrm{a}$ shows the percentage of participants in each group who met the cut-off criterion of significant decline on three or more tests in study 1 and two or more tests in study 2. Approximately $39 \%$ of both the hormonal and chemotherapy group met criterion as compared to $7 \%$ of the healthy control in both studies (light gray column). Figure $2 b$ shows the effect of using a more stringent cut-off criterion-significant decline on four or more tests in study 1 and three or more tests in study 2 . Not surprisingly, the percentage of participants meeting this more stringent criterion decreases to $24 \%$ in the hormonal group and $20.5 \%$ in the chemotherapy group. None of the healthy controls are identified using this criterion.

Table 5 provides a comparison of individual participants' performance between our previous study and the current one. In the hormonal group, $56.5 \%$ of the participants met the cut-off criteria of significant decline on three or more tests using the long battery. Of these, $39 \%$ were also identified by the reduced screening battery, while 
Table 4 Descriptive statistics of groups

\begin{tabular}{|c|c|c|c|c|}
\hline & Healthy control & Hormonal group & Chemotherapy group & $p$ value \\
\hline Age at baseline ${ }^{a}$ & $59.4(4.1)$ & $57.5(4.2)$ & $57.5(3.9)$ & .088 \\
\hline Range & $51-66$ & $50-65$ & $50-66$ & \\
\hline Education at baseline $^{\mathrm{a}}$ & $16.0(2.5)$ & $14.0(2.9)$ & $14.4(3.0)$ & .014 \\
\hline Range & $12-21$ & $9-20$ & $8-23$ & \\
\hline Estimated IQ at baseline & $46.0(2.4)$ & $44.0(3.1)$ & $44.7(3.1)$ & .013 \\
\hline Range & $40-50$ & $35-50$ & $36-49$ & \\
\hline Test-retest interval $^{\mathrm{b}}$ & $151.9(30.1)$ & $158.9(31.4)$ & $144.6(34.9)$ & .107 \\
\hline Range & $98-218$ & $123-245$ & $91-245$ & \\
\hline
\end{tabular}

Mean values shown, with standard deviations in parentheses

$I Q$ Intelligence quotient as measured by the Quick Test (Ammons and Ammons 1962)

${ }^{a}$ In years

b In days

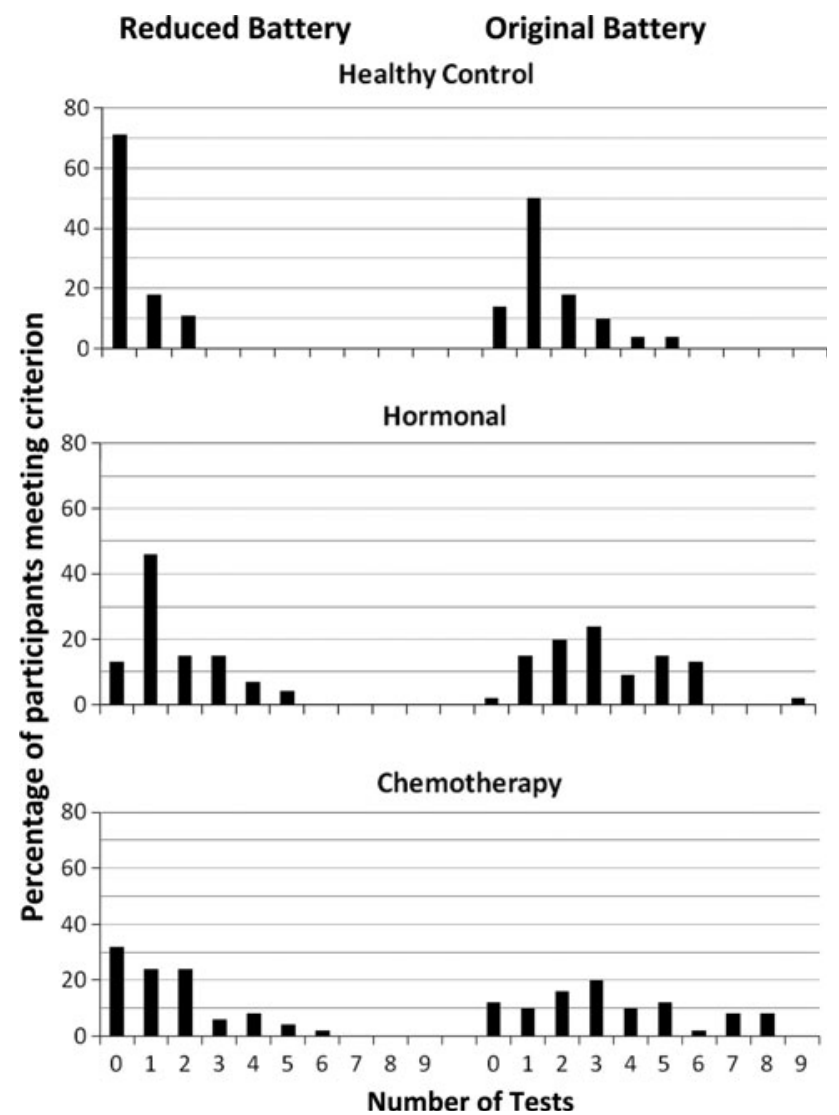

Fig. 1 Percentage of participants meeting criterion for cognitive decline on 0-9 tests based on comparison with a healthy control group. The left side of each graph presents the results from the reduced battery (study 2) and the right side, the comprehensive battery (study 1). The three horizontal panels represent the results of the healthy control, hormonal, and chemotherapy groups, respectively

$17.5 \%$ did not meet the cut-off criteria of decline on two or more tests on the same battery. One participant in this group did not meet the cut-off criterion for the larger battery, but was identified by the reduced battery. (a) Rates of decline using cut-off criterion 3 on S1 and 2 on S2

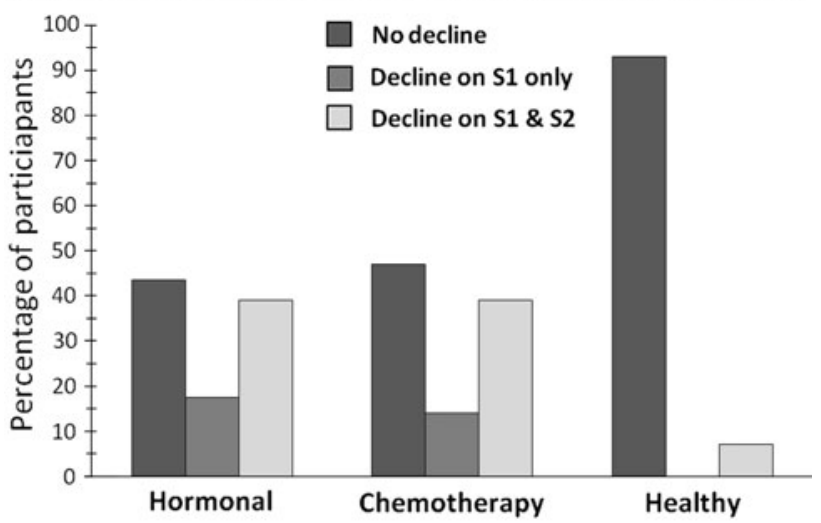

(b) Rates of decline using cut-off criterion 4 on S1 and 3 on S2

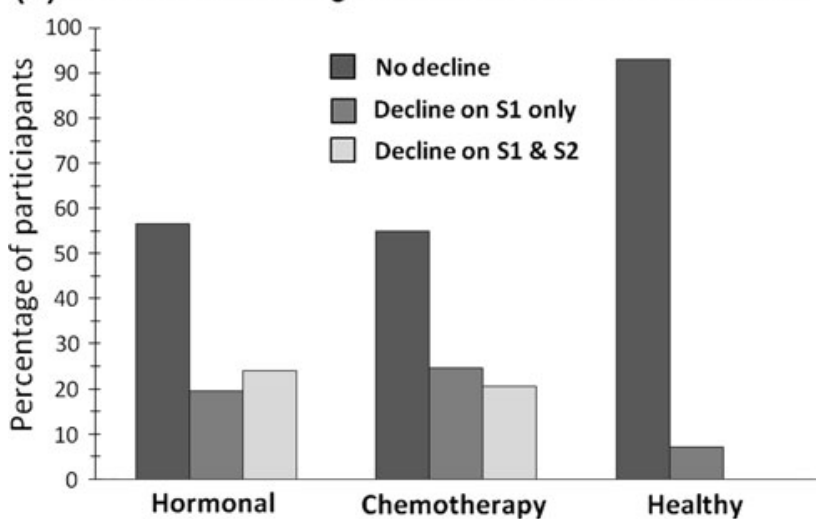

Fig. 2 Percentage of participants who met the cut-off criterion of significant decline of three or more tests in study 1 (S1) and two or more tests in study 2 (S2) are presented in upper panel (a). The lower panel (b) illustrates the percentage of participants who met a more stringent cut-off criterion of significant decline on four or more tests in S1 and three or more tests in S2

Results were similar in the chemotherapy group; $53 \%$ of participants met the cut-off criterion on the large battery, of which $39 \%$ were identified by the screening battery and 
Table 5 Comparison of individual decline from study 1 to study 2

\begin{tabular}{lllll}
\hline Participant & \# tests & $\%$ tests & \# tests & $\%$ tests \\
number & declined & $\begin{array}{l}\text { declined } \\
\text { study 1 }\end{array}$ & $\begin{array}{l}\text { declined } \\
\text { study 2 }\end{array}$ & $\begin{array}{l}\text { declined } \\
\text { study 2 }\end{array}$ \\
\hline
\end{tabular}

Hormonal group

259

253

251

250

246

244

241

240

237

230

228

223

219

217

216

212

207

204

$260^{\mathrm{a}}$

$256^{\mathrm{a}}$

$254^{\mathrm{a}}$

$248^{\mathrm{a}}$

$242^{\mathrm{a}}$

$236^{\mathrm{a}}$

$221^{\mathrm{a}}$

$208^{\mathrm{a}}$

$203^{\mathrm{b}}$

Healthy control group

$\begin{array}{ll}423 & 5 \\ 409 & 4 \\ 401^{\text {b }} & 2\end{array}$

Chemotherapy group

\begin{tabular}{lllll}
168 & 8 & 35 & 4 & 44 \\
165 & 4 & 17 & 2 & 22 \\
164 & 3 & 13 & 2 & 22 \\
157 & 3 & 13 & 2 & 22 \\
151 & 7 & 30 & 2 & 22 \\
149 & 7 & 30 & 4 & 44 \\
147 & 4 & 17 & 2 & 22 \\
145 & 5 & 22 & 5 & 55 \\
137 & 5 & 22 & 4 & 44 \\
136 & 5 & 22 & 2 & 22 \\
135 & 3 & 13 & 2 & 22 \\
131 & 4 & 17 & 4 & 44 \\
130 & 7 & 30 & 3 & 33 \\
120 & 8 & 35 & 3 & 33 \\
\hline
\end{tabular}

Table 5 continued

\begin{tabular}{lllll}
\hline $\begin{array}{l}\text { Participant } \\
\text { number }\end{array}$ & $\begin{array}{l}\text { \# tests } \\
\text { declined } \\
\text { study } 1\end{array}$ & $\begin{array}{l}\text { \% tests } \\
\text { declined } \\
\text { study } 1\end{array}$ & $\begin{array}{l}\text { \# tests } \\
\text { declined } \\
\text { study } 2\end{array}$ & $\begin{array}{l}\text { \% tests } \\
\text { declined } \\
\text { study } 2\end{array}$ \\
\hline $\mathbf{1 1 9}$ & $\mathbf{8}$ & $\mathbf{3 5}$ & $\mathbf{6}$ & $\mathbf{6 6}$ \\
$\mathbf{1 1 8}$ & $\mathbf{4}$ & $\mathbf{1 7}$ & $\mathbf{2}$ & $\mathbf{2 2}$ \\
$\mathbf{1 1 6}$ & $\mathbf{7}$ & $\mathbf{3 0}$ & $\mathbf{5}$ & $\mathbf{5 5}$ \\
$\mathbf{1 1 4}$ & $\mathbf{3}$ & $\mathbf{1 3}$ & $\mathbf{2}$ & $\mathbf{2 2}$ \\
$\mathbf{1 0 2}$ & $\mathbf{6}$ & $\mathbf{2 6}$ & $\mathbf{3}$ & $\mathbf{3 3}$ \\
$163^{\mathrm{a}}$ & 5 & 22 & 1 & 11 \\
$162^{\mathrm{a}}$ & 4 & 17 & 1 & 11 \\
$159^{\mathrm{a}}$ & 4 & 17 & 1 & 11 \\
$158^{\mathrm{a}}$ & 8 & 35 & 1 & 11 \\
$128^{\mathrm{a}}$ & 5 & 22 & 0 & 0 \\
$121^{\mathrm{a}}$ & 5 & 22 & 1 & 11 \\
$105^{\mathrm{a}}$ & 5 & 22 & 1 & 11 \\
$140^{\mathrm{b}}$ & 2 & 9 & 2 & 22 \\
$127^{\mathrm{b}}$ & 2 & 9 & 2 & 22 \\
$124^{\mathrm{b}}$ & 2 & 9 & 2 & 22 \\
\hline
\end{tabular}

Bold numbers indicates participants showing decline on 3 or more tests in study 1 and identified by the reduced battery in study 2

${ }^{\text {a }}$ Participants showing decline on 3 or more tests in study 1 but not identified by the reduced battery in study 2

b Participants showing decline on fewer than 3 tests in study 1 but identified by the reduced battery in study 2

$14 \%$ did not meet criterion. Of the $47 \%$ of participants in the chemotherapy group who did not meet the cut-off criterion on the large battery, $6 \%$ (three participants) were still identified by the reduced battery.

In the healthy control group, only two participants (7\%) showed significant decline on four or more tests in study 1 , and they were also identified by the reduced battery in study 2 . One additional participant who did not meet the long battery cutoff criterion was identified by the reduced battery.

\section{Discussion}

The goal of this study was to compare the sensitivity of a small battery of neuropsychological tests to detect significant decline in cognitive function following chemotherapy treatment with that of a comprehensive battery. Comparisons were made using a healthy control group as a reference and two criteria levels. The results were similar in that comparable numbers of participants were identified by both batteries, suggesting that individuals vulnerable to chemo-fog can be identified as easily by a more selective battery emphasizing specific domains. This has a great clinical utility.

Nevertheless, there were some participants (roughly $19 \%$ ) who were identified by the comprehensive battery and not the reduced battery using both the relaxed and 
more stringent criterion. This could be a result of the cutoffs being too low although there were reasonable statistical grounds for the selected values. Another possibility is that certain tests are more sensitive to age-related cognitive decline than the effects of chemotherapy.

There are certain limitations to our current study. Declines in function in this population may not be entirely attributable to chemotherapy. Various other explanations have been proposed, including disease-related stress, fatigue and depression. Although our study found no evidence that fatigue or depressive symptoms played a role in performance (i.e., non-significant as covariates) they should still be routinely evaluated.

Some researchers have remarked that baseline assessments are often undertaken at a time of great stress and may therefore not provide an accurate comparison point post-treatment. This is useful to bear in mind because if baseline scores are underestimated due to stress, then the degree of subsequent impairment will also be underestimated, and apparent improvement may be overestimated.

Adjuvant hormonal therapies may also lead to cognitive declines. In fact, although we are attempting to develop a screening tool that is sensitive to the cognitive deficits specifically associated with chemotherapy, the results suggest that the hormonal group meets screening criterion almost as frequently as the chemotherapy group, despite having received no chemotherapy treatment. One possible explanation for this is differing patterns of tests results such that chemotherapy patients may be declining on a distinct combination of tests; therefore, the battery may need to be modified to reflect the declines more particular to chemotherapy treatment versus hormonal therapies. Current research also suggests that there are cognitive impairments associated with hormonal treatment [31-33] and it may be difficult to distinguish these effects from those resulting from chemotherapy alone.

In this study, our comparison was a healthy control group as opposed to a hormonal or another treatment group. It has been demonstrated that the nature of the control group can influence the quality of analysis [34] and that careful matching ensures that pre-existing factors do not overly influence the results. Based on this observation, it could be argued that our healthy control group does not share the characteristics associated with having cancer. However, given the aim of the study, underestimation of decline and misclassification were considered to be of primary importance and the healthy control group was considered the best comparison group for decline in function from a healthy state.

Lastly, there was a statistically significant difference in education and estimated IQ between the healthy control group and the two treatment groups, suggesting the healthy control group may have more cognitive reserve; this could account for some of the differences observed between groups. However, it should be noted that based on standardized IQ scores (as measured by WAIS instruments), all participants fell within \pm one standard deviation from the mean (i.e. $100 \pm 15$ ). With respect to education, approximately two-thirds of the hormonal and chemotherapy participants completed post-secondary studies, as compared to slightly over half of the healthy control participants.

Despite these significant differences at a group level, when the covariates were evaluated at an individual level using the SRB analyses, they only accounted for a significant proportion of the variance for one of the nine variables, suggesting that they are not exerting a great influence on the majority of decline scores.

Although several of the limitations in neuropsychological assessment described in the introduction cannot be readily addressed in this paper-lack of clean constructs, fixed versus flexible batteries, limited theory-there are some that can be effectively minimized. These include: redundancy and misclassification and issues with poor psychometrics, which can be dealt with through the use of a reduced battery and careful selection of tests based on available empirical resources and research. Professional guidelines or policies would also be very beneficial to instruct clinicians on the most effective and reliable means of constructing a neuropsychological test battery, and would reduce test selection based solely on availability, tradition, familiarity, or personal preference.

Finally, studies evaluating subjective measures of cognitive impairment in recipients of chemotherapy and hormonal therapy are still very limited. This type of research will be important in determining which aspects of cognitive functioning have the most negative impact on quality of life for the breast cancer survivor. Further work is also still needed to clarify which neuropsychological tests are most sensitive to detecting cognitive decline associated with chemotherapy treatment so that assessment batteries can be limited to fewer tests. Preliminary research in this area has identified a limited number of tests that appear to be sensitive to declines in this population $[10,29]$ but more research of this nature must be done in order to improve clinical efficiency and reduce the likelihood of misclassification.

Acknowledgments We gratefully acknowledge the women who participated in this study, without whom our research could not be conducted. We also want to thank the Canadian Breast Cancer Foundation for their generous funding of our work.

Conflict of interest There are no conflicts of interest in relation to this article.

\section{References}

1. Ouimet LA, Stewart A, Collins B, Schindler D, Bielajew C (2009) Measuring neuropsychological change following breast 
cancer: an analysis of statistical models. J Clin Exp Neuropsychol 31:73-89

2. Canadian Cancer Society (2010) Breast cancer statistics. http://www.cancer.ca/Canada-wide/About $\% 20$ cancer/Cancer\%20 statistics/Stats\%20at\%20a\%20glance/Breast\%20cancer.aspx?sc_ lang=en. Accessed 11 Sept 2010

3. American Cancer Society (2010) How many women get breast cancer? http://www.cancer.org/Cancer/BreastCancer/Overview Guide/breast-cancer-overview-key-statistics. Accessed 11 Sept 2010

4. Abraham J, Haut MW, Moran MT, Filburn S, Lemiuex S, Kuwabara H (2008) Adjuvant chemotherapy for breast cancer: effects on cerebral white matter seen in diffusion tensor imaging. Clin Breast Cancer 8:88-91

5. Collins B, Mackenzie J, Stewart A, Bielajew C, Verma S (2008) Cognitive effects of chemotherapy in post-menopausal breast cancer patients 1 year after treatment. Psychooncology 18:134-143

6. Debess J, Riss JO, Engebjerg MC, Ewertz M (2010) Cognitive function after adjuvant treatment for early breast cancer: a population-based longitudinal study. Breast Cancer Res Treat 121:91-100

7. Ferguson RJ, Ahles TA, Saykin AJ, McDonald BC, Furstenberg CT, Cole BF (2007) Cognitive-behavioral management of chemotherapy-related cognitive change. Psychooncology 16: 772-777

8. Hermelink K, Untch M, Lux MP, Kreienberg R, Beck T, Bauerfeind I, Munzel K (2007) Cognitive function during neoadjuvant chemotherapy for breast cancer. Cancer 109:1905-1913

9. Hurria A, Lachs M (2007) Is cognitive dysfunction a complication of adjuvant chemotherapy in the older patient with breast cancer? Breast Cancer Res Treat 103:259-268

10. Jansen CE, Miaskowski CA, Dodd MJ, Dowling GA (2007) A meta-analysis of the sensitivity of various neuropsychological tests used to detect chemotherapy-induced cognitive impairments in patients with breast cancer. Oncol Nurs Forum 34:997-1005

11. Jim HSL, Donovan KA, Small BJ, Andrykowski MA, Munster PN, Jacobsen PB (2009) Cognitive functioning in breast cancer survivors: a controlled comparison. Cancer 115:1776-1783

12. Kesler SR, Bennett FC, Mahaffey ML, Spiegel D (2009) Regional brain activation during verbal declarative memory in metastatic breast cancer. Clin Cancer Res 15:6665-6673

13. Reid-Arndt SA, Yee A, Perry MC, Hsieh C (2009) Cognitive and psychological factors associated with early posttreatment functional outcomes in breast cancer survivors. J Psychosoc Oncol 27:415-434

14. Stewart A, Collins B, Mackenzie J, Tomiak E, Verma S, Bielajew C (2008) The cognitive effects of adjuvant chemotherapy in early stage breast cancer: a prospective study. Psychooncology 17:122-130

15. Tager FA, McKinley PS, Schnabel FR, El-Tamer M, Cheung YK, Fang Y, Golden CR, Frosch ME, Habif U, Mulligan MM, Chen IS, Hershman DL (2010) The cognitive effects of chemotherapy in post-menopausal breast cancer patients: a controlled longitudinal study. Breast Cancer Res Treat 123:25-34

16. Taillibert S, Voillery D, Bernard-Marty C (2007) Chemobrain: is systemic chemotherapy neurotoxic? Curr Opin Oncol 19:623-627

17. Ahles TA, Saykin AJ, Furstenberg CT, Cole B, Mott LA, TitusErnstoff L, Skalla K, Bakitas M, Silberfarb PM (2005) Quality of life of long-term survivors of breast cancer and lymphoma treated with standard-dose chemotherapy or local therapy. J Clin Oncol 23:4399-4405

18. Downie FP, Mar Fan HG, Tchen A, Yi Q, Tannock IF (2006) Cognitive function, fatigue, and menopausal symptoms in breast cancer patients receiving adjuvant chemotherapy: evaluation with patient interview after formal assessment. Psychooncology 15:921-930

19. Fallowfield LJ, Leaity SK, Howell A, Benson S, Cella D (1999) Assessment of quality of life in women undergoing hormonal therapy for breast cancer: validation of an endocrine symptom subscale for the FACT-B. Breast Cancer Res Treat 55:189-199

20. Kayl AE, Wefel JS, Meyers CA (2006) Chemotherapy and cognition: effects, potential mechanisms, and management. Am J Ther 12:362-369

21. Mar Fan HG, Houede-Tchen N, Yi Q, Chemerynsky I, Downie FP, Sabate K, Tannock IF (2005) Fatigue, menopausal symptoms, and cognitive function in women after adjuvant chemotherapy for breast cancer: 1 and 2 year follow-up of a prospective controlled study. J Clin Oncol 23:8025-8032

22. Mehnert A, Scherwath A, Schirmer L, Schleimer B, Peterson C, Schulz-Kindermann F, Zander A, Koch U (2007) The association between neuropsychological impairment, self-perceived cognitive deficits, fatigue and health related quality of life in breast cancer survivors following standard adjuvant versus high-dose chemotherapy. Patient Educ Couns 66:108-118

23. Von Ah D, Russell KM, Storniolo AM, Carpenter JS (2009) Cognitive dysfunction and its relationship to quality of life in breast cancer survivors. Oncol Nurs Forum 36:326-336

24. Weis J, Poppelreuter M, Bartsch HH (2009) Cognitive deficits as long-term side-effects of adjuvant therapy in breast cancer patients: 'subjective' complaints and 'objective' neuropsychological test results. Psychooncology 18:775-782

25. Wong TM (2006) Ethical controversies in neuropsychological test selection, administration, and interpretation. Appl Neuropsychol 13:68-76

26. Strauss E, Sherman E, Spreen O (2006) A compendium of neuropsychological tests: administration, norms, and commentary, 3rd edn. Oxford University Press, New York

27. McSweeney AJ, Naugle RI, Chelune GJ, Lunders H (1993) "T scores for change": an illustration of a regression approach to depicting change in clinical neuropsychology. Clin Neuropsychol 7:300-312

28. Lezak MD, Howieson DB, Loring DW (2004) Neuropsychological assessment, 4th edn. Oxford University Press, New York

29. Freeman JR, Broshek DK (2002) Assessing cognitive dysfunction in breast cancer: what are the tools? Clin Breast Cancer 3:591-599

30. Lucas J, Addeo RR (2005) Traumatic brain injury and postconcussive syndrome. In: Snyder PJ, Nussbaum PD (eds) Clinical neuropsychology: a pocket handbook for assessment, 2nd edn. American Psychological Association, Washington, DC, pp 351-380

31. Bender CM, Sereika SM, Berga SL, Vogel VG, Brufsky AM, Paraska KK, Ryan CM (2006) Cognitive impairment associated with adjuvant therapy in breast cancer. Psychooncology 15:422-430

32. Castellon SA, Ganz PA, Bower JE, Peterson L, Abraham L, Greendale GA (2004) Neurocognitive performance in breast cancer survivors exposed to adjuvant chemotherapy and tamoxifen. J Clin Exp Neuropsychol 26:955-969

33. van Dam FS, Schagen SB, Muller MJ, Boogerd W, Wall E, Droogleever Fortuyn ME, Rodenhuis S (1998) Impairment of cognitive function in women receiving adjuvant treatment for high-risk breast cancer: high-dose versus standard-dose chemotherapy. J Natl Cancer Inst 90:210-218

34. Sawrie SM (2002) Analysis of cognitive change: a commentary on Keith et al. (2002). Neuropsychology 16:429-431 